\title{
Clonality analysis in lymphoproliferative disease using the BIOMED-2 multiplex PCR protocols: experience from the EuroClonality group EQA scheme
}

\author{
Susan Harris • M. Bruggemann • P. J. T. A. Groenen • \\ E. Schuuring $\cdot$ A. W. Langerak $\cdot$ E. Hodges
}

Received: 16 February 2012 / Accepted: 17 February 2012 / Published online: 14 March 2012

(C) Springer-Verlag 2012

\begin{abstract}
The BIOMED-2 primers and PCR protocols for the detection of clonal proliferations in lymphoproliferative disease are well established and are used in many Molecular Pathology laboratories worldwide due to their availability as commercial kits. The kits have made the technical performance of these protocols relatively uncomplicated; however, the technical interpretation is less straightforward, since the reporting of these assays requires knowledge of TCR and BCR gene recombinations, multiple rearrangement patterns and cross-lineage rearrangements. The initial challenge was to first standardise the analysis of these assays across
\end{abstract}

S. Harris $(\bowtie) \cdot$ E. Hodges

Department of Molecular Pathology,

University Hospital Southampton NHS Foundation Trust,

Duthie Link building, Mail Point 225, Tremona Road,

Southampton SO16 6YD, UK

e-mail: sue.harris@uhs.nhs.uk

\section{Bruggemann}

Second Medical Department,

Universitätsklinium Schleswig-Holstein,

Campus Kiel,

Kiel, Germany

\section{P. J. T. A. Groenen}

Department Pathology, Nijmegen Medical Centre,

Radboud University,

Nijmegen, The Netherlands

\section{E. Schuuring}

Department of Pathology, University Medical Centre,

Erasmus MC,

Groningen, The Netherlands

A. W. Langerak

Department of Immunology, Erasmus MC,

Rotterdam, The Netherlands
Europe via the EuroClonality group and to issue guidelines for the analysis, interpretation and reporting of the BIOMED-2 assays. This has been achieved and this standardized scoring system will be adopted as an essential part of the BIOMED-2 EuroClonality EQA scheme.

Keywords Euroclonality EQA Scheme - Clonality analysis interpretation $\cdot$ Lymphoproliferative disease

\section{Introduction}

The molecular identification of clonal T-cell (TCR) and Bcell (BCR) receptor gene rearrangements has proved to be a useful addition in the classification and diagnosis for difficult cases of lymphoproliferative disease [1]. The European BIOMED-2 collaborative study designed new TCR and immunoglobulin (Ig) primers in 18 multiplex PCR reactions, which have now become well established as reliable assays, with high levels of sensitivity and detection rates for many lymphoproliferative diseases [2]. The standardisation of these multiplex PCR protocols led to their availability in commercial kits (Invivoscribe Technologies, San Diego, CA), which are now used routinely by molecular laboratories worldwide. Since the reporting of these clonality assays requires knowledge of TCR and Ig gene recombinations and multiple rearrangement patterns, and an awareness of the nonspecific amplifications and cross-lineage rearrangements, a robust external quality assessment (EQA) scheme is needed to assure the correct performance and accurate interpretation of results in laboratories performing these assays. The ultimate challenge is to standardise the EQA for clonality analysis across Europe and, via the 
EuroClonality group, to issue guidelines for the analysis, interpretation and reporting of the EuroClonality/BIOMED2 assays.

Participation in EQA is a valuable opportunity for laboratories to compare their performance against that of their peers and against agreed standards of practice [3]. Ideally, an EQA scheme should challenge the whole pre-analytical, analytical and post-analytical process. The EuroClonality group is comprised of more than 20 laboratories with considerable technical experience of the BIOMED-2 primers and protocols. They provided a unique opportunity to assess the analytical and post-analytical process for these protocols and therefore produce guidelines for the interpretation and reporting of clonality analysis results. It was decided not to include a check of the pre-analytical phase for the EQA scheme at this time.

\section{Aims}

1. Analyse the interpretation of GeneScan (GS) and polyacrylamide heteroduplex (HD) gel results of BCR and TCR clonality analysis

2. Develop a scoring system-common language for describing profiles

3. Define good standards using these protocols (best practice)

4. Highlight technical pitfalls and interpretation problems

5. Interpret EQA results into a quality score which can be used for accreditation

\section{Materials and methods}

Starting in the summer of 2008 until the summer of 2011, the Euroclonality group organised five EQA rounds to test the performance of BIOMED-2 based clonality assessment and EQA rounds within the group are still ongoing. For the 5 EQAs, DNA extracted from different haematological and histological cases, to include both GS and HD analysis (Table 1) were used. In addition, interpretation of data files for GS analysis and photographic images of HD gels was assessed. Cases were selected by the individual trial organiser to include both classical lymphoproliferations as well as more difficult cases. In earlier trials, each case was tested using all 18 of the BIOMED-2 multiplex PCR tubes. In addition, a process of developing a standardized technical description (scoring system) for reporting these results from each individual multiplex tube was initiated.

Further trials were then used to refine and standardise these technical descriptions (standardized scoring system) and to introduce an overall 'Molecular interpretation' for
TCR and BCR loci reporting. DNA provided from diagnostic cases, together with relevant clinical/phenotypic information, was then analysed using selected BIOMED-2 tubes to test the applicability of this standardized scoring system. Each centre processed the DNA according to their routine diagnostic PCR workflow dependent on the suspected diagnosis. Centres were asked to return results for the individual loci, an overall molecular interpretation and finally a clinical interpretation, if appropriate. For EQA trial 5, the revised standardized scoring system was finally tested using 50 consecutive routine diagnostic cases by each participating centre.

\section{Results}

Trial 1 -organiser Kiel

This first EQA round was technically oriented to establish the QC network focussing on the analytical phase of BIOMED-2 based molecular diagnostics. Molecular pathology conclusions were not requested in this QC round.

DNA from six fresh frozen lymphoproliferation samples (2 T-cell neoplasms, 2 B-cell neoplasms, and 2 reactive samples; Table 2) were sent out to all interested institutes of the original BIOMED-2 group [1]. The tasks were (1) to test the integrity of DNA using the BIOMED-2 control primers and (2) to amplify each DNA with all BIOMED-2 tubes being used in the respective lab. PCR products were evaluated using GS and/or HD analysis.

Twenty-three centres participated in this 'wet trial'. The results were discussed at the BIOMED-2/Euroclonality meeting in Kiel in September 2008. After a first general presentation of the results, three smaller discussion groups were organised to discuss the major findings. Their conclusions were finally summarized later during the meeting.

All centres assessed DNA quality with the BIOMED-2 primers, however some centres only used a part of the control gene primers (one centre only used the 600-bp primer set stating that a positive 600 -bp result suffices to affirm a good DNA quality, five centres did without the $600-\mathrm{bp}$ PCR product because DNA amplifying products greater than 300-bp was deemed suitable for further clonality analysis [4]).

For analysis of the BIOMED-2 Ig/TCR PCR products, 19 centres performed GS analysis, 14 centres did HD analysis, 10 of them in addition to GS (Table 2). Eight centres performed the whole Ig/TCR panel, 15 only used subsets depending on application within routine workflow of the respective centre. However, some centres also included tubes only for educational reasons with primer sets not being used in their daily routine and therefore not being validated in their centre. 


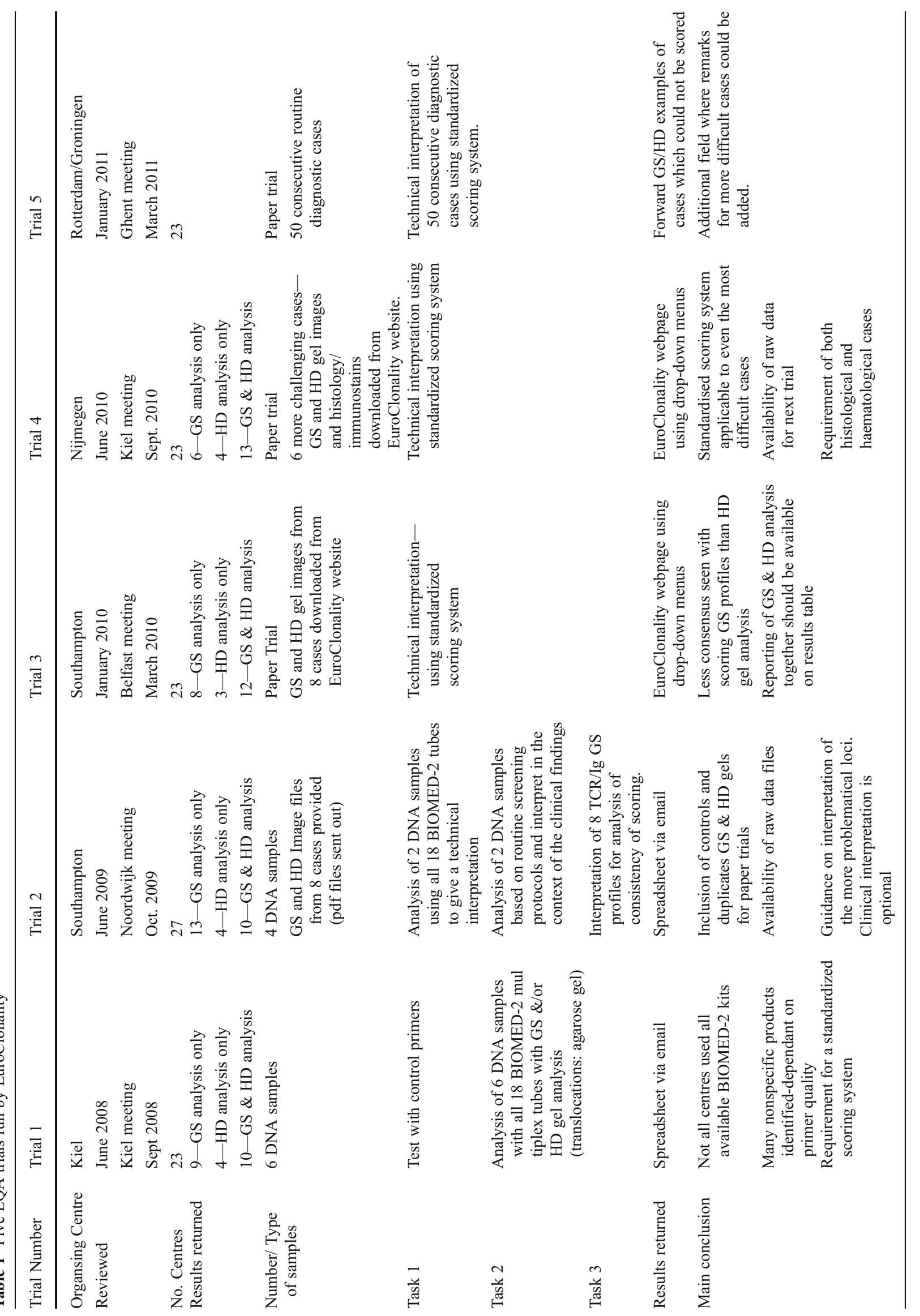


Table 2 For trial 1, tasks 1 and 2, DNA for 6 cases with the following diagnoses were provided

\begin{tabular}{lll}
\hline Case & Tissue & Diagnosis (not provided) \\
\hline 1 & Lymph node & Lymphadenitis \\
2 & Blood & T lymphoblastic leukaemia \\
3 & Lymph node & Chronic lymphocytic leukaemia/ small lymphocytic lymphoma \\
4 & Lymph node & Diffuse large B-cell lymphoma, not otherwise specified \\
5 & Lymph node & Lymphadenitis \\
6 & Bone marrow & T lymphoblastic leukaemia \\
\hline
\end{tabular}

Most cases showed concordant results, discrepancies mainly concerned the differentiation between clonality and nonspecific bands outside the expected size range. Many of these nonspecific bands particularly occurred in situations with a limited number of target genes (Ig targets in case 2 and 6, TCR targets in case 3 and 4) and had not fully been previously published [1]. The frequency of these nonspecific peaks was higher when analysis was performed by GS compared to HD. Their sizes (in base pairs) partly differed depending on whether centres used their own in-house BIOMED-2 multiplex primer mixes or the BIOMED-2 Invivoscribe kits.

An additional major finding of this EQA was the problematic comparability of data reporting (e.g. reporting of oligoclonality or nonspecifc products). It was therefore concluded that a universal description of the BCR/TCR clonality testing data was essential to make any scoring system workable and to allow a standardized interpretation of results. It was decided that a first proposal of this universal description should be presented during the next meeting. To focus on this problem, it was also decided to include a "paper task" with provided GS and HD images to test the consistency of such a description system.

As this first trial was not scored, each centre was given a certificate of participation.

\section{Trial 2-organiser Southampton}

Twenty-seven centres participated in this second EQA round which involved both an analytical and interpretation elements, grouped into three tasks. The results were discussed at the BIOMED-2/EuroClonality meeting in Noordwijk in June 2009. As per the previous trial the results were presented and smaller discussion groups were organised to discuss the major findings.

\section{Task 1}

This involved testing DNA from two cases, with a clinical and phenotypic diagnosis for B-cell lymphocytic leukaemia (B-CLL) and T-cell prolymphocytic leukaemia (T-PLL), with all 18 of the BIOMED-2 primer mixes.
Both cases showed concordant results for the identification of clonal BCR gene rearrangements in B-CLL, and clonal TCR gene rearrangements for the T-PLL cases. However, less concordance was seen for the TCR loci in the BCLL case and the BCR loci in the T-PLL case. This may reflect the low lymphocyte numbers for these loci, as both cases had a high percentage tumour load. Many centres reported nonspecific peaks, especially in loci where there were limited lymphocyte numbers - thus highlighting the need to avoid the over interpretation of GS profiles and the necessity to test samples in duplicate. A second method of analysis could also be useful to confirm unusual GS profiles. Discussions also noted that the preferred methods of analysis, i.e. HD or GS analysis should be emphasized in more detail for loci such as Ig light chains and IgH (D-J) analysis [1].

\section{Task 2}

This involved DNA from a T-PLL and marginal zone lymphoma (MZL) cases. Each centre was asked to use their routine molecular diagnostic protocols, based on the clinical information provided, to provide a technical interpretation for each locus tested, an overall TCR/BCR technical molecular interpretation and finally a clinical interpretation in the context of the available diagnostic information.

For both cases, consensus was seen for the identification of the clonal TCR rearrangements in the T-PLL and BCR rearrangements in the MZL cases, although routine PCR screening protocols varied between different centres. Fifty-eight percent of centres tested various combinations of TCR beta (TCRB), gamma (TCRG) and delta (TCRD) gene rearrangements for the T-cell case, whereas $42 \%$ of centres tested various combinations of TCR and Ig gene rearrangements.

When testing for suspected B-cell lymphoproliferations, $50 \%$ of centres tested various combinations of Ig gene rearrangements only and 50\% performed various combinations of both Ig and TCR gene rearrangements.

Thirty percent of centres returned results for a technical interpretation only and an overall technical interpretation for all loci was returned by $62 \%$ of the centres. Fifty-four percent of the centres also interpreted these finding with 
the clinical information provided based on their normal practice.

Small group discussions confirmed that the reporting of a clinical interpretation for this EQA scheme should be optional, as this practice is dependent on the context of the laboratory performing the molecular testing, the local responsibility of a laboratory and also on the additional clinical information provided, which cannot be quality-controlled.

\section{Task 3}

This task was to investigate the possibility of running a 'dry/ paper' trial.

GeneScan profiles from 8 cases (Table 3) were provided for centres to test the consistency of pattern identification for each profile.

Consensus was seen for the majority of loci showing clear poly/clonal GS profiles, however some loci were problematical. Ig kappa (IGK)-Tube A, Ig lambda (IGL), TCRB-Tube A, TCRG-Tubes A and B showed the greatest discrepancy for the interpretation of GS profiles. The most concordant loci included IgH tubes A, B and C, and TCRB tube $\mathrm{C}$.

The discussion groups highlighted difficulty with the interpretation of other centres data. They suggested duplicate GS images and supplementary clonal, polyclonal and negative control data for comparison with the EQA cases. Centres who normally only perform HD analysis requested the inclusion of gel images too. These requests would be addressed in the next QC trial along with the possibility of sending raw data files for individual analysis investigated.

\section{Trial 3-organiser Southampton}

Twenty-three centres participated in the third paper EQA round which involved a technical interpretation using the standardized scoring system proposed from three previous

Table 3 For trial 2, task 3 the GeneScan profiles for 8 cases with the following diagnosis were provided

\begin{tabular}{ll}
\hline Case & Diagnosis provided with GS profiles \\
\hline 5 & Clonal TCR gamma/delta lymphocytosis \\
6 & Polyclonal control DNA \\
7 & Oligoclonal T-cell case \\
8 & B-cell non-Hodgkins lymphoma (B-NHL) \\
9 & Sezary syndrome \\
10 & Bi-clonal B-CLL \\
11 & Reactive lymphocytosis \\
12 & Bi-clonal B-cell disease \\
\hline
\end{tabular}

trials. The results were discussed at the BIOMED-2/EuroClonality meeting in Belfast March 2010. The results were presented before smaller discussion groups were organised to discuss the major findings.

Image files for eight diagnostic cases (Table 4) were downloaded by each centre from the Euroclonality website (www.EuroClonality.org), and included duplicate GS profiles and HD gel images for each loci plus clonal, polyclonal and negative controls. The technical analysis of individual loci and the overall technical interpretations were returned via the website by selecting drop-down options using the scoring system proposed from previous trials.

Consensus was seen with clearly poly/clonal TCR/BCR profiles, the IgH locus demonstrating the best concordance with the majority of cases. The exception was the T-PLL case (case 6), which had previously been shown to have very low B-cell numbers by phenotypic analysis. The TCR loci were less concordant, particularly in cases demonstrating complex TCR profiles (such as the oligoclonal T-LGL (case 1), the bi-phenotypic cases (case 3), and the B-CLL case (case 8) which had previously been shown to contain low T-cell numbers by phenotypic analysis).

The majority of centres recorded their GS and HD analysis separately. Less concordant results were returned for the TCR GS profiles when compared to the TCR HD gel analysis. Discussion groups highlighted the difficulty in describing some of the T-cell profiles at the technical level especially in B-cell disease. The standardisation of HD analysis may facilitate interpretation but should also be performed in duplicate.

\section{Trial 4-organiser Nijmegen}

The aim of this paper trial was to test the adapted standardized coding system from the previous trials using six more challenging cases (Table 5), based on their complex rearrangement profiles in genescanning. In this trial, the clinical information and the GS and HD results data (all in duplicate) were downloaded from the EuroClonality website. Also, the GS raw data files were available via the website, allowing the participants to analyse the profiles according to their normal routine procedure. Histology and immunostainings of the cases were provided via the website as well. Participating pathologists could scroll over slides, enabling them to determine the tumour load and the representativity of the tissue samples.

Twenty-five centres participated in this trial and scored data files of all the Ig/TCR data that were provided. Two of the laboratories only scored subsets of PCR targets (scoring the BCR clonality and not the TCR data, or scoring of the IGH (FR) and the TCRG data solely). The results were returned via the web page. 
Table 4 For trial 3, duplicate GS profiles and HD gel images were provided from eight cases with brief phenotypic or histological data

\begin{tabular}{|c|c|c|c|c|}
\hline Case & Tissue & Phenotyping/histology & & Final diagnosis (not provided) \\
\hline 1 & Peripheral blood & $\begin{array}{l}\mathrm{WCC}=17.9 \times 10^{9} / \mathrm{L} \\
\mathrm{Lymph}=7.9 \times 10^{9} / \mathrm{L}\end{array}$ & $\begin{array}{l}\mathrm{CD} 3=7.4 \times 10^{9} / \mathrm{L} \\
\mathrm{CD} 19=0.24 \times 10^{9} / \mathrm{L}\end{array}$ & $\begin{array}{l}\text { CD3/8+ oligoclonal T-LGL } \\
\text { (T-cell large granulocytic leukaemia) }\end{array}$ \\
\hline 2 & Lymph node & \multicolumn{2}{|c|}{$\begin{array}{l}\text { Replacement of lymph node architecture by } \\
\text { polymorphous B-cell lymphocytes } \\
\text { Numerous T-cells present }\end{array}$} & Marginal zone lymphoma \\
\hline 3 & Skin biposy & \multicolumn{2}{|c|}{$\begin{array}{l}\text { B-cell population-CD20+, CD } 79 a+\text {, IgKappa }+ \\
\text { T-cell component - CD } 3 / 5+, \text { CD } 4+ \\
\text { Null cell population-CD30+ }\end{array}$} & Bi-phenotypic cutaneous lymphoma \\
\hline 4 & Peripheral blood & $\begin{array}{l}\mathrm{WCC}=7.0 \times 10^{9} / \mathrm{L} \\
\mathrm{Lymph}=2.5 \times 10^{9} / \mathrm{L}\end{array}$ & $\begin{array}{l}\mathrm{CD} 3=2.2 \times 10^{9} / \mathrm{L} \\
\mathrm{CD} 19=0.1 \times 10^{9} / \mathrm{L}\end{array}$ & Sezary syndrome \\
\hline 5 & Lymph node & \multicolumn{2}{|l|}{$\begin{array}{l}\text { Numerous T-cell blasts } \\
\text { Ki67 } 60 \%\end{array}$} & Anaplastic large cell lymphoma (Alk neg) \\
\hline 6 & Peripheral blood & $\begin{array}{l}\mathrm{WCC}=236 \times 10^{9} / \mathrm{L} \\
\mathrm{Lymph}=226 \times 10^{9} / \mathrm{L}\end{array}$ & $\begin{array}{l}\mathrm{CD} 3+/ 4+/ 8+=79 \% \\
\mathrm{CD} 19=0.0 \%\end{array}$ & T-cell prolymphcytic leukaemia \\
\hline 7 & Lymph node & \multicolumn{2}{|c|}{$\begin{array}{l}\text { Some preservation of architecture with } \\
\text { infiltrating neoplastic cells }=\text { CD30+, } \\
\text { CD4+, CD23+, CD3-, CD5-, CD7- }\end{array}$} & Classical Hodgkins disease \\
\hline 8 & Peripheral blood & $\begin{array}{l}\mathrm{WCC}=20 \times 10^{9} / \mathrm{L} \\
\mathrm{Lymph}=6.7 \times 10^{9} / \mathrm{L}\end{array}$ & $\begin{array}{l}\text { CD } 3=68 \% \\
\text { CD } 19=28 \%, \text { Igkappa }=23 \%\end{array}$ & B-cell chronic lymphcytic leukaemia \\
\hline
\end{tabular}

Participants were not provided with a final diagnosis at this stage

This trial demonstrated the importance of experience with the normal GS patterns, the interpretation of isolated clonal targets and knowledge of rare gene recombinations. Case 1 was a morphologically unusual Hodgkin lymphoma or T-cell lymphoma. An isolated clonal IGK-DE rearrangement was identified on both GeneScanning and Heteroduplex analysis, which was unnoticed and missed by centres that only scored the IGH-PCRs. Clearly, the molecular interpretation was that clonality of the Kappa light chain was detected. From the pathological point of view showing morphological features and expression of the B-cell transcription factor Pax 5 by the neoplastic cells is suggested a diagnosis of Hodgkin's lymphoma. The monoclonal rearrangement is compatible with this diagnosis.
Case 2 was a lymph node biopsy in which EBER-positive B-cells and CD8-positive T-cells were detected. The differential diagnosis either was a CD8-positive T-cell proliferation in the context of an EBV infection or a T-cell lymphoma. This case generated discussion on how to report cases where the GS and HD analysis do not support each other. The IGH-rearrangement patterns on GeneScanning showed small seemingly dominant peaks on top of a Gaussian curve, which were not detectable by Heteroduplex analysis. The majority of the laboratories scored these patterns as polyclonal. Two of three clonal TCR rearrangements were detected on Genescanning but not by HDA because of the low intensity signal. On the other hand, a TCRB rearrangement was only detected by HDD and not by the

Table 5 For trial 4, duplicate GeneScan profiles and heteroduplex gel images for 6 more challenging cases were provided with the histological data and immunocytochemical findings and a working diagnosis

\begin{tabular}{|c|c|c|c|c|}
\hline Case & Tissue & Histochemistry & Histology images & Working diagnosis \\
\hline 1 & Lymph node & $\begin{array}{l}\text { Tumour cells } \\
\text { CD30+, CD45+, CD4+, CD2-, } \\
\text { CD3-, CD5-, CD20-, CD79a- }\end{array}$ & H\&E, PAX 5 & Unusual Hodgkins or T- cell lymphoma \\
\hline 2 & Lymph node & $\begin{array}{l}\mathrm{EBV}+\mathrm{B} \text {-cells } \\
\mathrm{CD} 8+\text { T-cells }\end{array}$ & $\mathrm{H} \& \mathrm{E}, \mathrm{CD} 3$ & $\begin{array}{l}\text { CD8+ T-cell proliferation in the contex } \\
\text { of EBV or T-cell lymphoma }\end{array}$ \\
\hline 3 & Lymph node & $\begin{array}{l}\text { Large interfollicular B-cell proliferation } \\
\text { with high proliferation in T-cell area }\end{array}$ & $\mathrm{H} \& \mathrm{E}$ & B-cell lymphoma or reactive lesion \\
\hline 4 & Post mortem & CD8, CD20, EBER & $\mathrm{H} \& \mathrm{E}$ & Splenomegaly? lymphoma \\
\hline $5 \mathrm{a}$ & Lymph node 2009 & $\mathrm{CD} 20+$ & $\mathrm{H} \& \mathrm{E}$ & ?Recurrent B-cell lymphoma \\
\hline $5 b$ & Lymph node 2003 & As above & As above & Diagnostic sample \\
\hline
\end{tabular}


GeneScan prints presented. The majority of the centres scored the clonal rearrangements and concluded this case showed clonal TCR rearrangements in a polyclonal background of T-cells, a picture that would fit with a CD8+ Tcell lymphocytosis in the context of EBV as could be seen in the provided slides. This case nicely underlies that clonality does not always mean malignancy. Based on the rearrangement patterns of this case, it was agreed that reproducible clonality signals detected either by GeneScanning or by Heteroduplex analysis are sufficient to report clonality.

Another case highlighted the existence of rare TCRG rearrangement detected in the size range of the $\mathrm{Vg} 11$ rearrangements (case 3 ) and that these results should be confirmed by sequence analysis (this case has been described in paper; L Groenen et al., manuscript in preparation).

For case 4, the control gene amplification identified poor quality DNA. Half of the centres reported this as 'results not interpretable due to low DNA quality', whereas the other half of the centres reported clonality. Although the sample was of poor quality, clonality could be confirmed: reproducible clonal signals were obtained in multiple PCR targets by GeneScanning, and some of the targets were even detectable by HDA. The reproducibility of the "clonal" signals even at low signal intensity, is of crucial importance, as was shown later by confirming a small reproducible clonal peak to be a true rearrangement.

The last case was a comparison of two biopsies from one patient to see whether there was recurrence of disease. The clonal signals in both tissue biopsies, with high tumour load were convincing. The scoring of these cases was fully concordant.

\section{Trial 5-organiser Rotterdam/Groningen}

The aim of this trial was to evaluate the uniform scoring system for final approval by all centres. Participants were asked to score 50 consecutive routine diagnostic cases from their laboratories, excluding samples for minimal residual disease target identification or somatic hypermutation analysis (for B-CLL). The total number of cases analysed was 1150 (756 B-cell requests and 621 T-cell requests). Overall only 36 cases $(3.1 \%)$ were scored as 'difficult to interpret' using the scoring system. This trial also highlighted the mixture of all types of material analysed in many of the different laboratories around Europe.

The discussion groups identified that centres were able to use the standardized scoring system to report the majority of their cases. Difficulties in applying the scoring system were seen more often for the TCR loci; however, these difficulties could be resolved by the addition of a field for remarks. Centres that only perform HD analysis highlighted that not all options in the standardized reporting were relevant and that problems were seen in cases where more than one clonal rearrangement was seen per tube.

\section{Discussion}

Standardisation of the BIOMED-2 primers and multiplex PCR protocols has led to their availability in commercial kits. These have made the technical performance of these protocols straightforward, allowing them to be used by many laboratories worldwide. However, the technical interpretation is less straightforward and best considered in two parts; firstly, reporting a technical description of each individual tube tested. Secondly, a molecular interpretation/conclusion, where all targets are considered together. This may be more easily achieved by reporting the B-cell and T-cell findings separately, although this does require a full knowledge of TCR/BCR gene recombinations to avoid misinterpretation of data. If a standardized scoring system could be devised, these post-analytical phases would be quite straightforward to evaluate by EQA.

The final part of the post-analytical process should ideally include a clinical interpretation of the molecular findings, interpreted with any histological, phenotypic and clinical data provided. However, for this EQA scheme, it was decided that this would be optional at this point, as this practice is dependent on the context of the laboratory performing the molecular testing, the local responsibility of a laboratory and also on the additional clinical information provided, which cannot be quality-controlled.

Ideally, an EQA scheme should challenge the whole preanalytical, analytical and post-analytical processes. The technical expertise of the EuroClonality group who had been involved in the design, validation and standardisation of the BIOMED-2 primers and protocols, simplified the development of this EQA scheme by allowing us to concentrate our initial efforts on the post-analytical process. We have devised standardized universal descriptions for the TCR and BCR clonality profiles which are applicable to the majority of routine diagnostic lymphoproliferative cases.

The initial aim of the EuroClonality group was to devise a standardized scoring system for the technical description of TCR/BCR clonality analysis using the BIOMED-2 primers and protocols. This standardisation has now been achieved and validated on 1,150 routine diagnostic cases by the 23 European centres of various disciplines (Haematopathology, Haematology and Immunology centres) participating in these trials. The aim was to be able to describe $95 \%$ of all cases using the standardized scoring system, which has been achieved as only $36(3.1 \%)$ cases were identified as difficult to score using the system. Cases where profiles could not be reported with the standard scoring system have been resolved by the addition of a field where a remark could be added. This 
standardized scoring system will be published and adopted as an essential part of the EuroClonality EQA scheme (Langerak et al., manuscript in preparation).

EuroClonality now has a reputable web site established in 2009 where EQA trial information and files can be downloaded and trial results returned (www.EuroClonality.org). The EuroClonality/BIOMED-2 consortium will continue to run EQA trials and work towards producing guidelines for the pre- and post-analytical phases for BIOMED-2 TCR/ BCR clonality testing in lymphoproliferative disease.

Acknowledgements To all centres who participated in these first five EQA trials

Molecular Pathology, University Hospital Southampton NHS Foundation Trust, Southampton, UK.

Dept. Immunology, Erasmus MC, Rotterdam, Netherlands.

Dept. Pathology, University MC Groningen, Groningen, Netherlands.

Dept. Pathology, Radboud University Nijmegen Medical Centre, Nijmegen, The Netherlands.

Dept. Pathology, University of Amsterdam, Medical Centre, Amsterdam.

Dept. Clinical Biology, Molecular Diagnostics, Heilig Hart Hospital, Roeselare, Belgium.

Dept. Hematology, University of Salamanca, Spain.

Dept. Haematology/Oncology, Institute Portuguese Oncology, Lisbon, Portugal.

Dept. Haematology-Oncology, Belfast, UK

Dept. Pathology, Frankfurt, Germany

II Medizinshe Klinik der Unversitat, Institute of Pathology, Kiel, Germany.

Institute of Pathology, Berlin, Germany.

Haematology Diagnostic Service, St James University Hospital Leeds, UK.

Dept. Medical Genetics, Aberdeen Royal Infirmary, Aberdeen, UK.

Dept. Hemato Oncology, The Institute of Cancer Research, Sutton Surrey, UK.
Dept. Histopathology, Addenbrooke's NHS Trust Hospital, Cambridge UK.

Dept. Pathology, UCL Medical School, London, UK..

Molecular Diagnostics, Nottingham City Hospital, Nottingham, UK.

Dept Pathology, Cardiff University, Cardiff, UK.

Molecular Genetics, Norfolk \& Norwich University Hospital, UK.

Hopital Necker-Enfants Malades, Paris, France:

Dept Genetic Oncology, Rouen, France.

Hopital Henri Mondor, Créteil, France

Dept. Pathology, Hopital Purpan, Toulouse, France.

Molecular Pathology Program, Centro Nacional de Investigaciones Oncologicas, Madrid, Spain.

Department of Human Pathology and Oncology, Siena, Italy.

University of Turin, Turin, Italy.

Dept. Pathology, Academic Hospital, Uppsala, Sweden.

Author declaration The authors declare they have no conflict of interest.

\section{References}

1. van Dongen JJM, Langerak AW, Bruggemann M et al (2003) Design and standardization for PCR primers and protocols for detection of clonal immunoglobulin and T-cell receptor gene recombinations in suspect lymphoporoliferations. Leukemia 17:22572317

2. van Krieken JHJM, Langerak AW et al (2007) Improved reliability of lymphoma diagnostics via PCR-based clonality testing: - Report of the BIOMED-2 Concerted Action BHM4-CT98-3936. Leukemia 21:201-237

3. Dequeker R, Ramsden D, Grody WW, Stenzel TT, Barton DE (2001) Quality control in molecular genetic testing. Nat Rev Genet 2(9):717-722

4. White HE, Lavender FL et al (2003) Use of BIOMED-2 protocols with DNA extracted from paraffin-embedded tissue biopsies and development of control gene primer set. Leukemia 17:2301-2304 